\title{
Chromophor Inhomogeneity Indication by Diffuse Vibronic Spectra
}

\author{
Vitaly Antonovich Tolkachev \\ Institute of Physics, National Academy of Sciences of Belarus, Minsk, Belarus
}

Email address:

tolkachev@ifanbel.bas-net.by

\section{To cite this article:}

Vitaly Antonovich Tolkachev. Chromophor Inhomogeneity Indication by Diffuse Vibronic Spectra. American Journal of Applied Chemistry. Vol. 8, No. 5, 2020, pp. 121-125. doi: 10.11648/j.ajac.20200805.11

Received: July 23, 2020; Accepted: August 5, 2020; Published: September 3, 2020

\begin{abstract}
Determination of gap between optically combining states (0-0- transition) from diffuse vibronic absorption or emission spectra is possible now for homogeneous ensembles of chromophores. If the observed spectra present composite polymorphic chromophores or different species they are formed by partial spectra and differing electronic transitions. For these conditions the indicating pure-electronic transition frequency attribute is distorted, smeared or even absent. That behavior is qualitative indication of the chromophore inhomogeneity. The same would be because of impurities. It is shown that the approach of inhomogeneity qualitative indication by spectra is adaptable to different structural forms of chromophor and at polymorphic sites of containing the chromophore media. The experimental data show that the approach is applicable to see the chromophore inhomogeneity by linear and circular vibronic spectra even of molecular dye-labels. The examples of observed distortions for the spectra of different composite species in different media as manifestation of their inhomogeneity are given. As the region of indication 0-0-transition is situated at low intensity antistokes wings of spectra the sensitivity to inhomogeneity is high as to hindrance by impurities and measurement precision.
\end{abstract}

Keywords: Optical Spectra, 0-0-Electronic Transition, Inhomogeneity of Chromophores, Spectral Inhomogeneity, Impurities Indication, Molecular Labels Homogeneity

\section{Introduction}

The application of basic quantum-theoretical microscopic reversibility principle to forming spectra mechanism of vibronic transitions with the thermal equilibrium in optical transition starting state have lead to new method of determining gap between combining in vibronic transition electronic states. The method was applied to molecular chromophores in vapor state and solutions [1-3], molecular crystals and quantum dots [4], F-centers and doped glasses [5] and molecular markers [4, 6]. Everywhere the homogeneous forms of chromophor gave single definite gap of states combining in electronic transition. For all the media the inhomogeneity was observed in different forms of development. It was observed evolution of doped glass inhomogeneity with time of annealing up to homogeneous [7] or transformation of diamond nanoparticles with annealing temperature from inhomogeneous form to homogeneous one [8]. Recently it was found that the method is applicable in natural and magnetically induced chirooptics
[9]. It was shown by this highly selective spectroscopy of complex molecules that integral linear spectra of the chiral substances often show lower homogeneity than chiral spectra. It is understandable.

\section{Theory}

It is found [1, 3] that for homomorphic chromophores in the vicinity of pure-electronic transition the crossection spectra $\sigma(v)$ on the frequencies $V$ obey relation:

$$
\sigma(v) \cdot \exp (\mp h v / k T)=\varphi\left(\left|v-v_{0}\right|\right)
$$

with "-" for absorption, "+" for emission; $h v_{0}$ is purely electronic gap between optically combining electronic states. The relation states symmetry around $\boldsymbol{v}_{0}$, extremum in the adjacent area. Function $\varphi$ prevails in the regions $v>v_{0}$ for absorption and $v<v_{0}$ for emission, i.e., is valid in the Stokes regions where the involvement of initial, starting in the 
transition sublevels is not limited by energy, but it extends to opposite side of $v_{0}$ on range of the thermal $k T$ order [4]. As the crossection $\sigma(v)$ in Eq. (1) is proportional to $\varepsilon(v) / v$ or $I(v) / v^{4}$ it is more convenient to use instead of (1) the usual absorption $\varepsilon(v)$ and emission spectra $I(v)$ in form:

$$
\ln (\varepsilon(v) / v)-h v / 2 k T=\ln \left(\varphi^{a}(v)\right)
$$

or

$$
\ln \left(I(v) / v^{4}\right)+h v / 2 k T=\ln \left(\varphi^{e}(v)\right)
$$

to analyze the location and form of $\varphi(v)$ extremum.

When the absorption coefficient $\varepsilon(v)$ at a given frequency $v$ is the sum of partial absorption coefficients $\varepsilon_{i}(v)$ of forms $i$ chromophores in the medium, (1) becomes as:

$$
\left[\sum_{i} \varepsilon_{i}(v) / v\right] \cdot \exp (-h v / 2 k T)=\sum_{i} \varphi_{i}\left(\left|v-v_{0 i}\right|\right)
$$

It is vivid that it is not symmetric like Eq. (1) and even may have no extremum if $v_{0 i}$ are not close $[4,6]$. Similarly, for emission intensity $I(v)$ it is

$$
\left[\sum_{i} I_{i}(v) / v^{4}\right] \cdot \exp (h v / 2 k T)=\sum_{i} \varphi_{i}^{e}\left(\left|v-v_{0 i}\right|\right) .
$$

The distorting of stating by (1), (2) or (3) extremum indicates that the chromophor is polymorphic according to (4) or (5) $[4,6]$. The situation by (4) and (5) is observed too when the spectra of different electron transitions of the same chromophores overlap. The problem is complicated and the way to the approach was considered particularly [3].
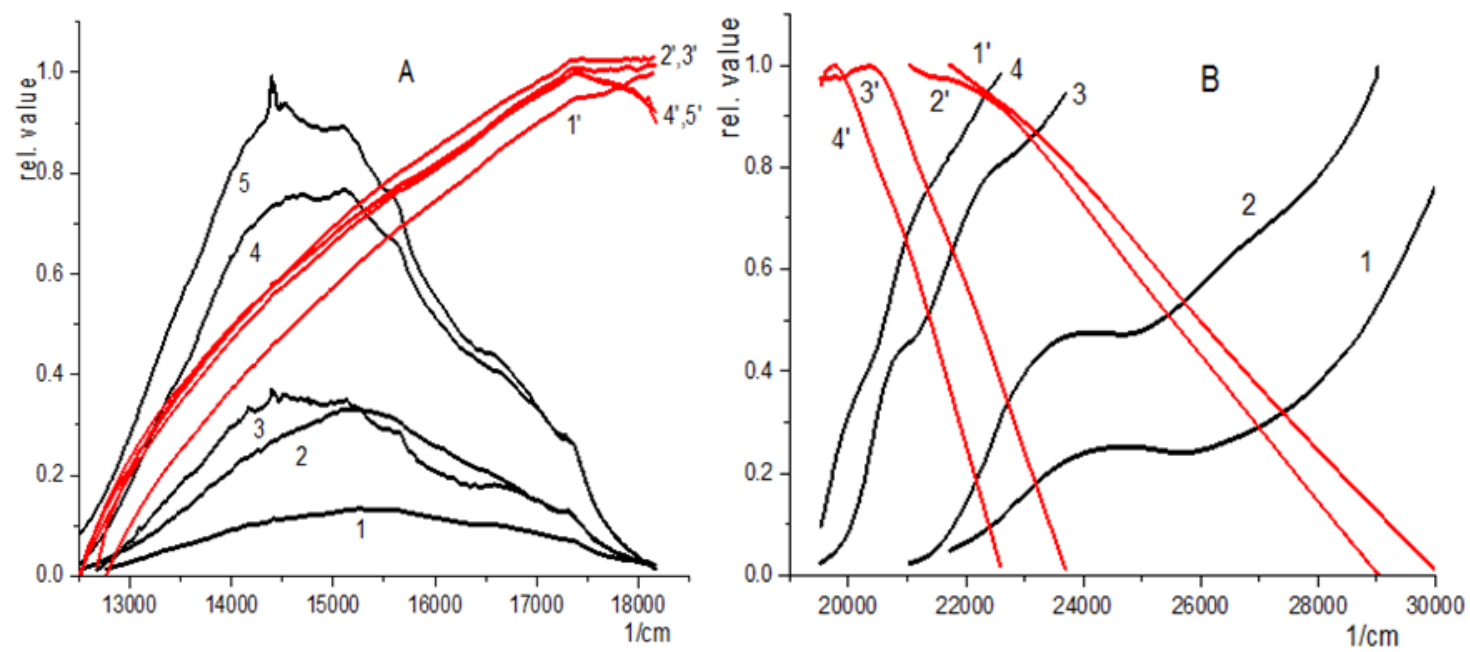

Figure 1. Transformation of micro object spectra along modification process. A are fluorescence spectra of diamond nanoparticles, obtained by oxidation in air with no annealing (1) and after annealing at temperatures $2-650^{\circ} \mathrm{C}, 3-700^{\circ} \mathrm{C}, 4-800^{\circ} \mathrm{C}, 5-900^{\circ} \mathrm{C}[8] ; 1^{\prime}-5^{\prime}$ are the corresponding $\ln \varphi^{e}$ (as usual throughout normalized in interval [0,1]) [4]. B are the change of absorption spectrum of glass doped with $C d S_{0.9} e_{0.1}$ crystallites after annealing at $650^{\circ} \mathrm{C}$ for 1 (1), 4 (2), 8 (3), and $16 \mathrm{~h}$ (4) [7] and the corresponding evolution of $\ln \varphi^{a}$ (1'- 4') [5].

\section{Application}

For example, absorption spectra of vapor state uracil and 1.3-dimethyluracil were compared in that way [2]. Their $v_{0}$ was determined by jet-cool spectroscopy and theoretical calculations. Uracil does not show extremum. The explanation is the well-known extensive tautomerism (up to seven tautomers) that persists to the temperature of the given spectrum $\left(211^{\circ} \mathrm{C}\right)$. Methyl substituents of 1,3-dimethyluracil prevent the tautomerism so $v_{0}$ determined by this and jet-cool methods were practically the same. Compounds allowing tautomerism did not exhibit 0 -0-transition extremum in the gas phase according to our observations. In a similar manner, the methyl substituents prevented tautomerism. For example, 1,3-dimethylhypoxanthine in the gas phase $\left(200^{\circ} \mathrm{C}\right)$ by it's UV absorption spectrum exhibits a well-resolved 0-0transition extremum about $32900 \mathrm{~cm}^{-1}$. However, the $0-0$ transition extremum is missing for the absorption spectrum of 9-methylhypoxanthine vapor $\left(273^{\circ} \mathrm{C}\right)$ because the molecule tautomerism is activated at high temperatures. For example, that was observed for tautomerism behavior of uracil, adenine and hypoxanthine. The "anomalies" show the possibility to analyze chromophores inhomogeneity by this approach [4, 6], so have practical interest.

In Figure 1 are given examples of micro objects absorption and fluorescence spectra transformation along the processes of technical modification. In Figure 1A the processes of annealing of diamond nanoparticles are given [8]. It is seen that in the first stages at lower temperatures the extremum is absent. But for temperatures $800-900^{\circ} \mathrm{C}$ when the process of modification is finished the extremum and $v_{0}$ are fixed well. Figure 1B shows process transformation of glass doped by annealing. $\mathrm{CdS}_{0.9} \mathrm{Se}_{0.1}$ crystallites in time [7]. At first stages of annealing the chromophores of glass are polymorphous. The results are in full absence of extremums. After longer time of annealing the chromophores ordered homogeneously and $v_{0}$. is manifested. 
In Figure 2 the emission spectra of graphene quantum dots (GQD) obtained by two different technologies are given and homogeneity of structure is determined. Figure 2A shows excited by different light waves emission spectra of obtained by chemical synthesis GQDs C132 [10, 11]. The spectra differ. It is because, probably, participating of differently

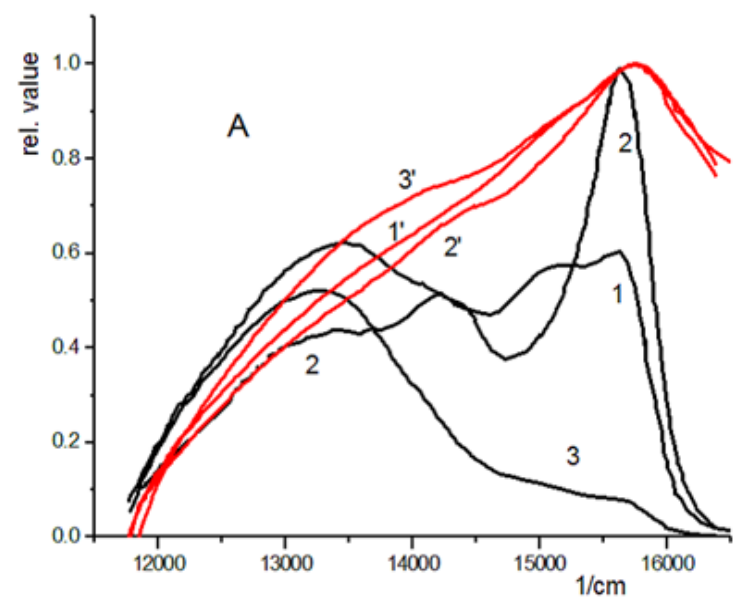

populated emitting levels. But upper emitting level is conserved showing the homogeneity. On Figure 2B are given GQDs spectra produced by mechanical-chemical method [12]. They are inhomogeneous by technology. There are emission spectra of three forms of chemically modified GQDs but no one have signs of homogeneity.

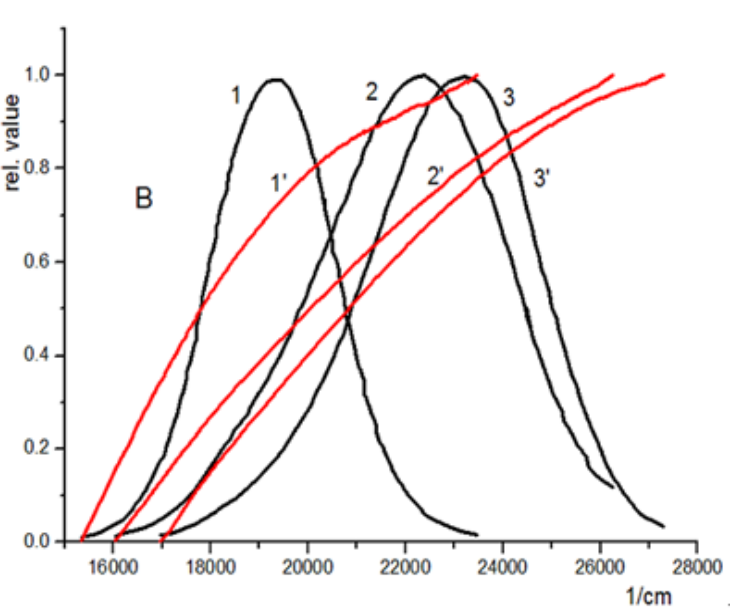

Figure 2. Emission spectra of GQD s: C132 obtained by chemical method: $1-\lambda_{\text {ex }}=337 \mathrm{~nm}, 2-\lambda_{\text {ex }}=479 \mathrm{~nm}, 3-\lambda_{\text {ex }}=532 \mathrm{~nm}[10,11$,$] (A), and of GQD s$ obtained by mechanical-chemical method: 1- non modified (oxido-GQDs), 2- previous ones doped by aminogrups (m-GQDs), 3- obtained from (m-GQDs) by reduction (r-GQDs) [12], (B); 1'-3' are the corresponding $\ln \varphi$ [4].
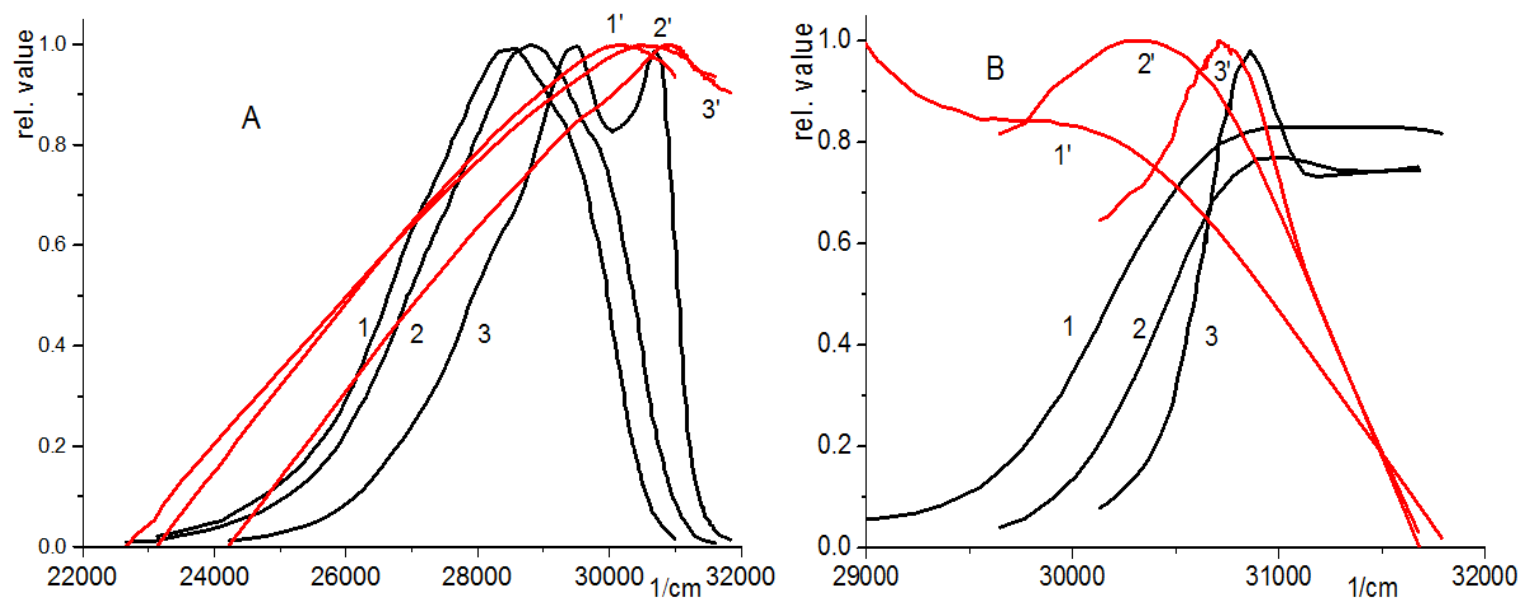

Figure 3. The fluorescence (A) and absorption (B) spectra of 2-dibenzofuranol in ethanol (1), acetonitrile (2) and cyclohexane (3) [13]; 1' -3'are corresponding $\ln \varphi$ spectra.

Let's view indication of molecular chromophores inhomogeneity. The examples of the composite extremes according (5) are given on Figure 3 for 2-dibenzofuranol [13]. The emission of ethanol, methanol and cyclohexane solutions in Figure 3A is presented by homogeneous excited state chromophores, perhaps of similar form. The opposite situation is for the ground state in Figure 3B. In cyclohexane solution for absorption $v_{0}$ is about $30700 \mathrm{~cm}^{-1}$, for emission about $30900 \mathrm{~cm}^{-1}$. For acetonitrile the values are 30350 and $30500 \mathrm{~cm}^{-1}$. (The difference for absorption and emission $v_{0}$ is the typical as related to prerelaxational finish-state, vertical zero-gap between combining states). The ground, absorbing state in ethanol solution is inhomogeneous, as has no extremum, but broad expanded to longwave side band. The effect one can explain by capability to form hydrogen bonding by ethanol which can produce polymorphic system of hydrogen-bonded chromophores.

Solvent on general have different interaction forms with solute up to produce the chemical complexes. In Figure 4A the indication of homogeneity transformation of chromophores solution in cyclohexane with addition of methanol [14] is presented by proposed method. The specific action of methanol on solute by hydrogen bonding is well known. The addition of methanol multiplies forms of solutesolvent cages and increase inhomogeneity. The behavior of common in biology aqueous solvents mostly depends on $\mathrm{pH}$. In Figure 4B the absorption spectra of deoxyadenosine at $\mathrm{pH}$ 1.5 and 7.9 are shown [15]. In alkali solution deoxyadenosine is homogeneous but in acidic solution the inhomogeneity, polymorphism is observed, as followed from deformation of characteristic function $\varphi$ spectrum. It is known that the 
alkali form of solution is preferable for homogeneity of many nucleotides, as has been confirmed by described method too [16].

Ultraviolet absorption (A) and magnetic circular dichroism (MCD) (B) spectra of oligopeptide antibiotics netropsin, distamycins $\mathrm{A}$ and $\mathrm{D}$ in aqueous $0.1 \mathrm{M} \mathrm{NaCl}$ solutions [17] are given in Figure 5. The application of 0-0-extremum method to the spectra show that MCD spectra gives clearly

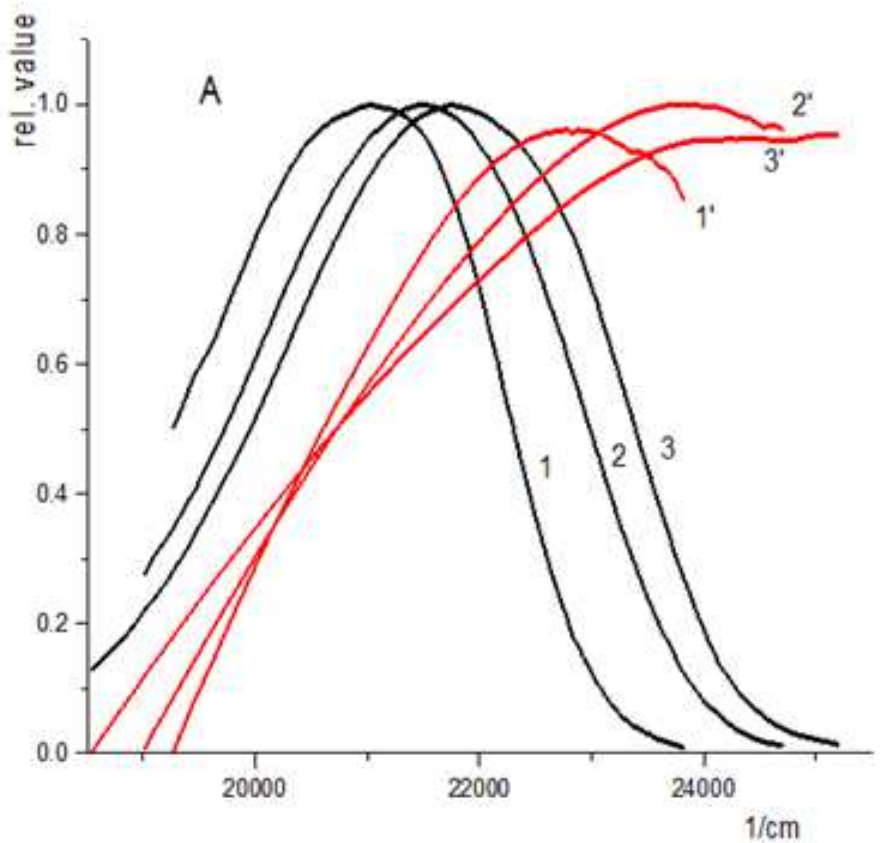

defined extremum $\left(v_{0}\right)$ and homogeneity. The corresponding points on plate A are shown by arrows. As the MCD spectra give clear $v_{0}$, so for integral absorption spectra, as noted by arrows, there are no extremums. The reason is that MCD spectra are spectra of chiral chromophores. The integral absorption spectra are spectra of general, may be even nonchiral structures and so are inhomogeneous.

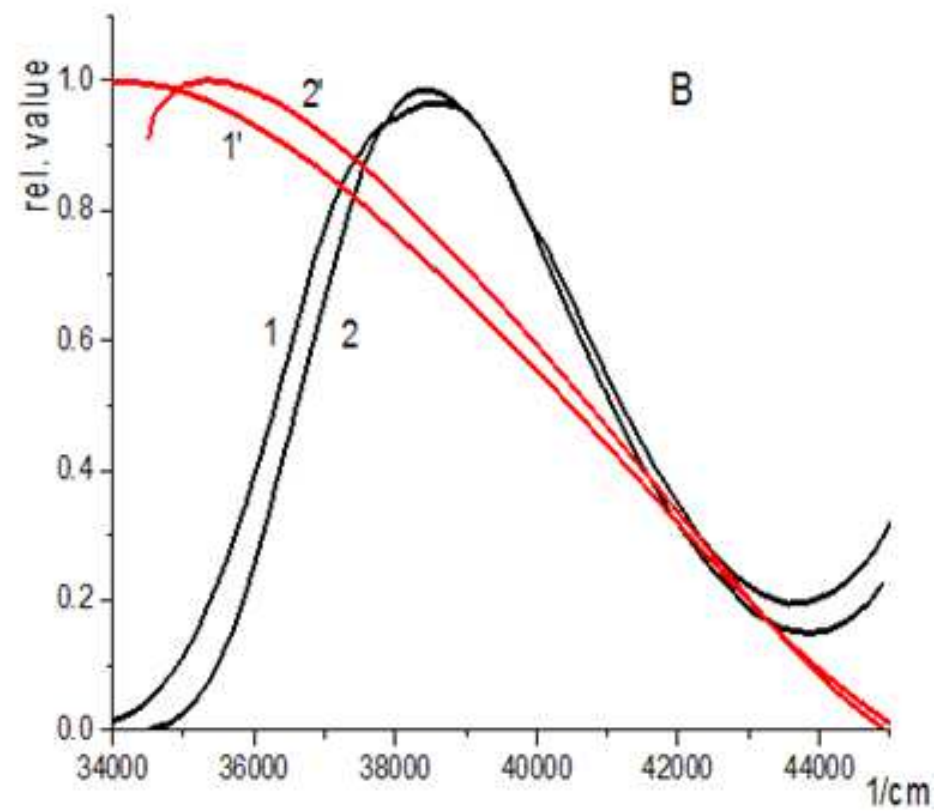

Figure 4. Influence of solvent on solute homogeneity shown by absorption and emission spectra. On plane A the fluorescence spectra of (2-hydroxyphenyl) benzimidazole in cyclohexane-methanol mixture: 1 no methanol (Me), $20.2 \mathrm{M} \mathrm{Me,} 30.4 \mathrm{M} \mathrm{Me} \mathrm{[14].} \mathrm{On} \mathrm{plane} \mathrm{B} \mathrm{absorption} \mathrm{spectra} \mathrm{of} \mathrm{aqueous}$ deoxyadenosine solution at $p H 1.5$ (1) and 7.9 (2) [15]. Corresponding $\ln \varphi$ are shown by primed numbers.
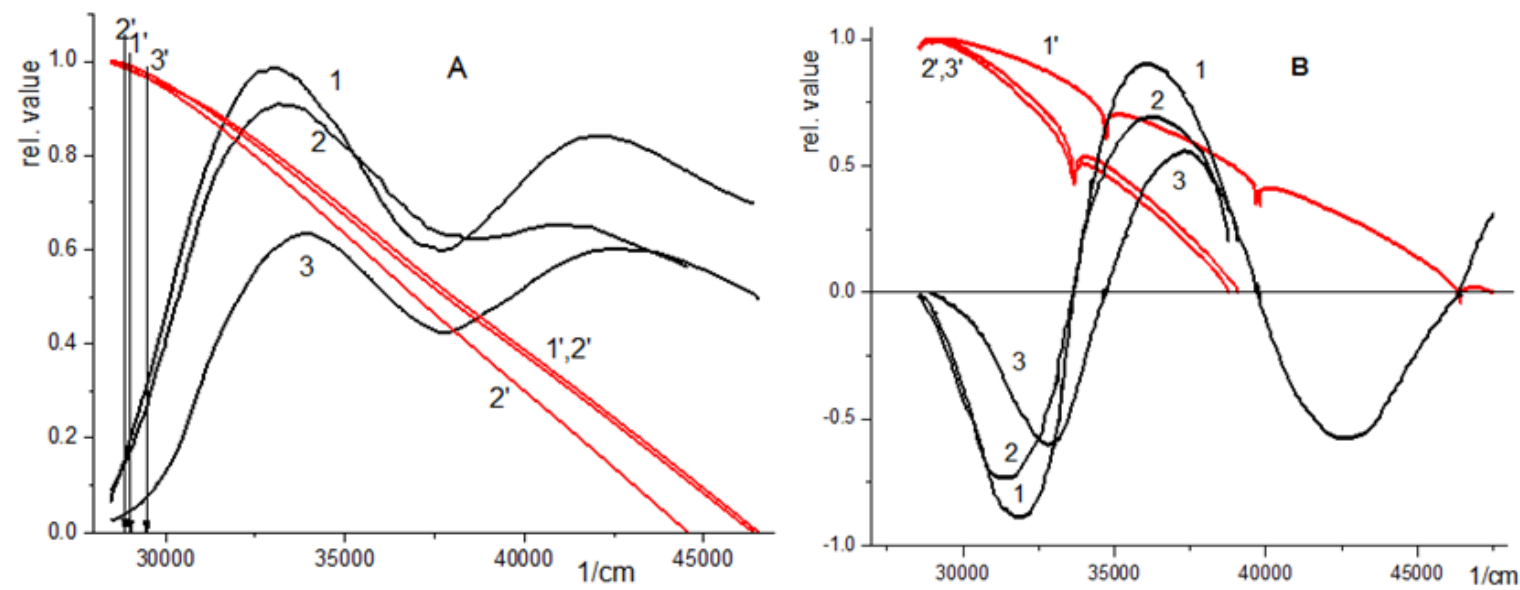

Figure 5. Ultraviolet absorption (A) and $M C D(B)$ spectra. Aqueous solutions of distamycin- $A$ in $\mathrm{H}_{2} \mathrm{O}$ (1); distamycin-D (2) and netropsin (3) in $0.1 \mathrm{M} N \mathrm{NaCl}$ spectra [17] and corresponding $\ln \left(\varphi^{a}\right)$ spectra $\left(1^{\prime}, 2^{\prime}, 3^{\prime}\right)$. The arrows on plate A note the positions of $v_{0}$ obtained in plate $B$.

\section{Conclusions}

It is shown that analysis of 0-0-transition localization can be effective to determine the spectral homogeneity of relative chromophor ensembles or even purity of species. It fits to gaseous, crystalline and polymeric media. The best application of the method beside gap $h v_{0}$ determination is to test homogeneity of molecular spectral labeling [3] and even of chromophor ensembles with chirooptical selection [17]. The main lack of the approach is location on low intensity antistokes wings of spectra, longwave in the absorption and short wave in the emission. So it is sensitive to overlapping spectra of different electronic transition and to measurement errors. The approach should be applied carefully at low temperatures [18]. 


\section{Acknowledgements}

The authors thank the Belarusian Republican Foundation for Fundamental Research for supporting the work

\section{References}

[1] V. A. Tolkachev (2017). Determination of 0-0-Transition Frequencies from Diffuse Vibronic Spectra. J. Appl. Spectrosc. 84, 668-673.

[2] V. A. Tolkachev (2017). Position of 0-0-Transition Frequency in Diffuse Vibronic Spectrum. Dokl. National. Acad. Sci. Belarus 61, 50-55 (rus.).

[3] V. A. Tolkachev (2018). Average Energies of Combining States and Purely Electronic Transition Frequencies in Vibronic Spectra. J. Appl. Spectrosc. 85, 845-849.

[4] V. A. Tolkachev and A. P. Blokhin (2019). Extraction of Purely Electronic Transition Frequency and Chromophor Polymorphism from Diffuse Vibronic Spectra. Sci. J. Anal. Chem. 7, 76-82.

[5] V. A. Tolkachev (2019). Zero-phonon Transition Frequency in Diffuse Electronic Spectra of Color Centers in Crystals and Glasses. J. Appl. Spectrosc. 86, 504-507, DOI 10.1007/s10812-019-00848-8.

[6] V. A. Tolkachev (2018). Manifestation of Molecular Chromophor Polymorphism in Diffuse Vibronic Spectra. J. Appl. Spectrosc. 85, 220-224.

[7] B. Andrianasolo, B. Champagnon, M. Ferrari, and N. Neuroth (1991). Nonlinear Effects in Microcrystalline Semiconductors. J. Lumin. 48-49, 306-308.

[8] X. Song, G. Wang, X. Liu, F. Feng, J. Wang, L. Lou and W. Zhu (2013). Generation of Nitrogen-Vacancy Color Center in Nanodiamonds by High Temperature Annealing. Appl. Phys. Lett. 102, 133109.

[9] V. A. Tolkachev (2020). Determining the Frequency of a
Purely Electronic Transition from Optical Activity Spectra. J. Appl. Spectrosc. 87 (3), 525-530.

[10] X. Yan, X. Cui and L. Li (2010). Synthesis of Large, Stable Colloidal Graphene Quantum Dots with Tunable Size. J. Amer. Chem. Soc. 132, 5944-5945.

[11] H. Riesen, Ch. Wieber and S. Schumacher (2014). Optical Spectroscopy of Graphene Quantum Dots: The Case of C132. J. Phys. Chem. A 118, 5189-5195.

[12] S. Zhu, J. Zhang, S. Tang, C. Qiao, L. Wang, H. Wang, X. Liu, B. Li, N. Yu, X. Wang, H. Sun and B. Yang (2012). Photoluminescence Mechanism in Graphene Quantum Dots: Quantum Confinement Effect and Surface/Edge State. Adv. Funct. Mater. 22, 4732-4740.

[13] P. R. Sainz-Rozas, J. R. Isasi and G. Gonzalez-Gaitano (2005). Spectral and photopysical properties of 2-dibenzofuranol and its inclusion complexes with cyclodextrin. J. Photochem. Photoboil. A: Chemistry 173, 319-327.

[14] E. L. Roberts, J. Dey and I. Warner. (1997). Excited-State Intramolecular Proton Transfer of 2-(2'-Hydroxyphenyl)benzimidazole in Cyclodextrins and Binary Solvent Mixtures. J. Phys. Chem. A 101, 5296-5301.

[15] D. Voet, W. B. Gratzer, R. A. Cox and P. Doty (1963) Absorption Spectra of Nucleotides, Polynucleotides, and Nucleic Acids in the Far Ultraviolet. Biopolymers 1, 193-208.

[16] V. A. Tolkachev (2020). New Opportunities for Analytical Use of Optical Diffuse Electronic Absorption and Emission Spectra. International Journal of Innovative Studies in Sciences and Engineering Technology 6 (5) 6-9.

[17] C. Zimmer, C. Marck, C. Schneider, D. Thiele, G. Luck, and W. Guschlbauer (1980). Magnetic Circular Dichroism Study of the Binding of Netropsin and Distamycin-A with DNA. Biochimica et Biophysica Acta 607, 232-246.

[18] V. A. Tolkachev (2020). Determination of the Pure-electronic Transition from Diffuse Absorption or Emission Spectra at Low Temperatures. J. Appl. Spectrosc. 87 (6) (Zh. Prikl. Spektrosk. 87 (6) (rus.)). (in print). 